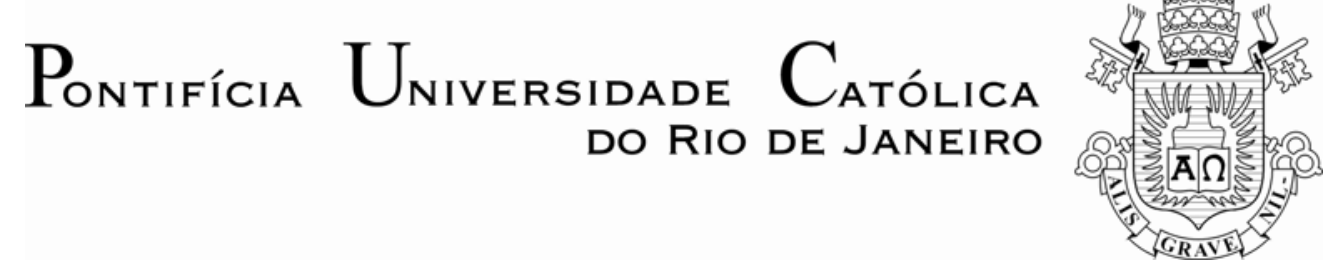

Thiago Broerman Cazes

Interpretação Baseada em Conhecimento de Imagens de Sensores Remotos de Alta Resolução

Dissertação de Mestrado

Dissertação apresentada ao Programa de Pósgraduação em Engenharia Elétrica da PUC-Rio como requisito parcial para a obtenção do título de Mestre em Ciências da Engenharia Elétrica

Orientador: Raul Queiroz Feitosa Co-orientador: Luiz Felipe Guanaes Rego

Rio de Janeiro, 09 de Setembro de 2005 


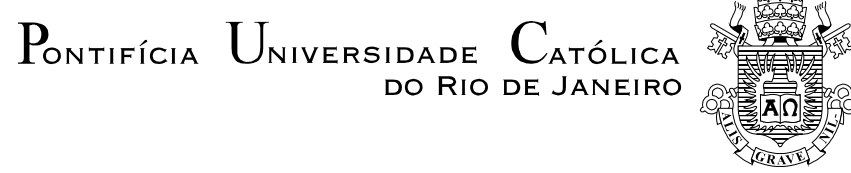

Thiago Broerman Cazes

\section{Interpretação Baseada em Conhecimento de Imagens de Sensores Remotos de Alta Resolução}

Dissertação apresentada como requisito parcial para obtenção do grau de Mestre pelo Programa de PósGraduação em Engenharia Elétrica do Departamento de Engenharia Elétrica do Centro Técnico Científico da PUCRio. Aprovada pela Comissão Examinadora abaixo assinada.

Dr. Raul Queiroz Feitosa Orientador

Departamento de Engenharia Elétrica - PUC-Rio

Dr. Luiz Felipe Guanaes Rego

Co-Orientador

Departamento de Geografia - PUC-Rio

Dra. Marley Maria Bernardes Rebuzzi Vellasco

Departamento de Engenharia Elétrica - PUC-Rio

Dr. Herman Johann Heinrich Kux

INPE

Prof. José Eugenio Leal

Coordenador Setorial do Centro

Técnico Científico - PUC-Rio

Rio de Janeiro, 09 de setembro de 2005 
Todos os direitos reservados. É proibida a reprodução total ou parcial do trabalho sem autorização da universidade, do autor e do orientador.

\section{Thiago Broerman Cazes}

Graduação em Engenharia Elétrica com Ênfase em Sistemas e Computação na UERJ. Objetivando título de Mestre em Métodos de Apoio a Decisão no Departamento de Engenharia Elétrica da PUC-RJ atuando em pesquisa na área de métodos automáticos de interpretação em sensoriamento remoto. Toma parte do projeto ECOWATCH que envolve instituições brasileiras e alemãs e é gerido pelo programa PROBRAL.

Ficha Catalográfica

Cazes, Thiago Broerman

Interpretação baseada em conhecimento de imagens de sensores remotos de alta resolução / Thiago Broerman Cazes ; orientador: Raul Queiroz Feitosa ; co-orientador: Luiz Felipe Guanaes Rego. - Rio de Janeiro : PUC, Departamento de Engenharia Elétrica, 2005.

94 f. ; $30 \mathrm{~cm}$

Dissertação (mestrado) - Pontifícia Universidade Católica do Rio de Janeiro, Departamento de Engenharia Elétrica.

Inclui referências bibliográficas.

1. Engenharia elétrica - Teses. 2. Sensoriamento remoto. 3. Imagens de alta resolução. 4. Interpretação de imagens baseada em conhecimento. 5. Classificação de imagens. 6. IKONOS. I. Feitosa, Raul Queiroz. II. Rego, Luiz Felipe Guanaes. III. Pontifícia Universidade Católica do Rio de Janeiro. Departamento de Engenharia Elétrica. VI. Título.

CDD: 621.3 


\section{Agradecimentos}

Ao meu orientador Prof. Raul Queiroz Feitosa;

Ao meu co-orientador Prof. Luiz Felipe Guanaes Rego;

A toda a equipe do projeto ECOWATCH;

Aos professores que participaram da Comissão examinadora;

Aos meus amigos da PUC-RIO;

A CAPES, ao DAAD e ao CNPQ pelo apoio financeiro;

Aos alunos de graduação Marlene, Daniele e Ricardo em geografia pelo apoio no procedimento de interpretação visual e apoio nos procedimentos específicos de cartografia;

Aos meus pais, familiares e amigos. 


\section{Resumo}

Cazes, Thiago Broerman; Feitosa, Raul Queiroz . Interpretação Baseada em Conhecimento de Imagens de Sensores Remotos de Alta Resolução Rio de Janeiro, 2005, 94 p. Dissertação de Mestrado - Departamento de Engenharia Elétrica, Pontifícia Universidade Católica do Rio de Janeiro.

A cada dia mais e mais satélites de alta resolução têm se tornado disponíveis, criando grande demanda por novos métodos de interpretação baseados em conhecimento. Estes métodos emulam parcialmente o trabalho do especialista em análise visual de imagens. Nesse contexto, o presente trabalho apresenta um modelo de classificação baseado no conhecimento do especialista aplicado a imagens de alta resolução. O modelo de interpretação consiste de três fases. A primeira inclui o conhecimento espectral e a informação de textura. Na segunda fase dados de SIG (sistema de informação geográfico) são combinados com o resultado da análise espectral através de regras nebulosas. Na terceira e última fase é introduzido o conhecimento multitemporal através de uma estimativa das possibilidades de transição entre classes de uma mesma área em um dado intervalo de tempo. Para validação desse modelo experimentos foram realizados em imagens IKONOS e fotos aéreas de 1999, 2001 e de 2002 da área do Parque Estadual da Pedra Branca, que é um importante fragmento da Floresta Atlântica situado no estado do Rio de Janeiro, Brasil.

\section{Palavras-Chaves}

Sensoriamento remoto; Imagens de alta resolução; Interpretação de imagens baseada em conhecimento; Classificação de imagens; IKONOS. 


\section{Abstract}

Cazes, Thiago Broerman; Feitosa, Raul Queiroz (Advisor). Knowledge-based Interpretation of High Resolution Remote Sensing Images Rio de Janeiro, 2005, 94 p. MSc. Dissertation - Department of Electrical Engineering, Pontifical Catholic

New high resolution satellites for commercial purposes became available in the few years. This increases the need of new automatic knowledge based image interpretation methods. Such methods partially emulate the reasoning of an image analyst during the visual image interpretation. The present work falls into this context and proposes an automatic classification model for high resolution remotely sensed images. The model consists of three stages. In the first stage only spectral and textural information are used for classification. In the second stage GIS (geographic information system) data are combined with the result of the spectral analysis by means of fuzzy rules. In the third stage the multitemporal knowledge is introduced by estimating class transition possibilities within a given time span. To validate the proposed model experiments were performed based on IKONOS images from 2001 and 2002 as well as aerial photos from 1999 of the Pedra Branca Park, which is an important Atlantic Forest fragment in the State Rio de Janeiro in Southeast Brazil.

\section{Keywords}

Remote sensing; High Resolution Images; Knowledge-based interpretation; Image Classification; IKONOS. 


\section{Sumário}

1 Introdução 13

1.1 Objetivos 15

1.1.1 Objetivo Geral 15

$\begin{array}{ll}1.1 .2 \text { Específico } & 16\end{array}$

$\begin{array}{lll}1.2 \text { Organização } & 16\end{array}$

2 Fundamentos Teóricos 17

$\begin{array}{ll}2.1 & \text { Sensoriamento remoto } \\ & 17\end{array}$

2.1.1 Sensoriamento remoto em imagens de alta resolução 19

2.1.2 Pré-processamento $\quad 22$

2.2 Métodos computacionais $\quad 24$

2.2.1 Classificação de padrões $\quad 24$

2.3 Segmentação 33

2.3.1 Histórico 33

2.3.2 Segmentação baseada em crescimento de regiões 34

2.3.3 Segmentação em múltiplas escalas 35

2.4 Parâmetros de textura 36

3 Estado da arte em classificação de imagens de alta resolução 37

3.1 Métodos baseados em pixels 37

3.2 Classificação baseada em objetos 40

3.3 Classificação Multitemporal 42

$4 \quad$ Modelo proposto $\quad 45$

4.1 Introdução $\quad 45$

4.2 Descrição geral do modelo 45

4.2.1 Fase 1: Classificação automática supervisionada baseada em dados espectrais e textura $\quad 47$

4.2.2 Fase 2: Classificação baseada em regras do especialista 47

4.2.3 Fase 3: Representação de conhecimento multitemporal 48

$5 \quad$ Avaliação experimental 51

5.1 Descrição da área de estudo e preparação dos dados 51

5.1.1 Parque Estadual da Pedra Branca 52

5.1.2 Imagens Utilizadas $\quad 56$

5.1.3 Preparação dos dados $\quad 58$

5.1.4 Segmentação $\quad 59$

5.1.5 Definição das classes $\quad 60$

5.1.6 Processo de classificação visual 64

5.2 Fase 1 - Classificação Espectral e Textura 68

5.2.1 Dados utilizados $\quad 68$

5.2.2 Resultados 69

5.3 Fase 2 - Classificação estrutural baseada em regras 70 
5.3.1 Atributos utilizados na classificação estrutural $\quad 71$

5.3.2 Regras 73

5.3.3 Ajuste das funções de pertinência

5.3.4 Resultados da classificação estrutural baseada em regras $\quad 75$

5.4 Fase 3 - Classificação multitemporal 77

5.4.1 Resultados do classificador multitemporal 82

5.5 Conclusões 86

$\begin{array}{lll}6 & \text { Comentários finais } & 87\end{array}$

$\begin{array}{lr}\text { Referências } & 89\end{array}$ 


\section{Lista de Figuras}

Figura 1 - Exemplo da influência da refletância e do comprimento de onda em diferentes tipos de cobertura de solo []

Figura 2 - resposta espectral relativa ao comprimento de onda na banda pancromática IKONOS [16]

Figura 3 - resposta espectral relativa, conforme o comprimento de ondas para o sensor multiespectral IKONOS [16]

Figura 4 - Representação do problema da topografia em captura de imagens de sensores remotos

Figura 5 - Modelo de neurônio artificial não linear [22]

Figura 6 - Funções de ativação: (a) tangente hiperbólica sigmóide (tansig) e (b) sigmóide logarítmica (logsig) a direita.

Figura 7 - Arquitetura de uma rede neural do tipo MLP com uma camada oculta $\quad 27$

Figura 8 - Representação de um individuo e genes em algoritmos genéticos 31

Figura 9 - demonstração do operador genético de crossover simples de um ponto. 32

Figura 10 - Modelo de interpretação baseado em conhecimento para imagens de alta resolução.

Figura 11 - diagrama detalhado do procedimento de interpretação na fase 2. 48

Figura 12 - Exemplo de diagrama de transição de estados para 4 classes

Figura 13 - Mapa representativo do Parque Estadual da Pedra Branca localizado no sul do Eestado do Rio de Janeiro.

Figura 14 - Imagem IKONOS Multiespectral (4m de resolução) da área do Parque Estadual da Pedra Branca no ano de 2001. Em azul o recorte correspondente a Figura 15 e Figura 16 mostrado apenas para exemplificar a riqueza de informação contida em imagens de alta resolução.

Figura 15 - Detalhe da área marcada em azul na Figura 14. Bandas visíveis (RGB). Observa-se a expansão urbana e as áreas de vegetação.

Figura 16 - Detalhe da área marcada em azul na Figura 14. Destaque na banda infravermelha. Composição (NRG) realçando a vegetação.

Figura 17 - Foto da área do Parque Nacional da Pedra Branca. Detalhe para a área da represa facilmente observável a partir da imagem de satélite. 
Figura 18 - Foto da área do Parque Nacional da Pedra Branca. Detalhe para a vegetação densa típica da Mata Atlântica.

Figura 19 - Foto do Parque Nacional da Pedra Branca; detalhe da expansão urbana sobre a área do parque. $\quad 56$

Figura 20 - Representação da imagem IKONOS 57

Figura 21 - Ilustração gerada com a foto aérea de 1999 e o DTM da área de estudo utilizado no processo de ortoretificação.

Figura 22 - Comparação entre as segmentações em uma área da imagem selecionada; a) imagem, segmentação com parâmetro de escala b) 90, c) 60 e d) 30 . 60

Figura 23 - Classificação Visual da imagem IKONOS de 2001. 


\section{Lista de Tabelas}

Tabela 1 - Características da legenda utilizada no processo de classificação.

Tabela 2 - Percentual de área na imagem classificada coberta por cada classe da legenda (imagens de 1999 e 2001 classificação visual)

Tabela 3 - Número de segmentos gerados por classe da legenda (imagens de 1999 e 2001, classificação visual)

Tabela 4 - Alterações reais detectadas pela classificação visual entre os anos de 2001 e 1999, relativo ao numero de segmentos.

Tabela 5 - Alterações reais detectadas pela classificação visual entre os anos de 1999 e 2001, percentual de mudança em relação ao ano de 1999.

Tabela 6 - Matriz de confusão da classificação espectral da imagem de 2001 após a primeira fase. Resultados apresentados em percentual relativo a imagem de referência.

Tabela 7 - Configuração dos sistemas neuro-fuzzy.

Tabela 8 - Matriz de confusão de uma classificação típica após a Fase 2. Resultado percentual relativo a referencia de uma interação.

Tabela 9 - Transições entre classes para a área de teste.

Tabela 10 - Configuração dos sistemas neuro-fuzzy.

Tabela 11 - Matriz de transição nebulosa. Valores em zero indicam transições impossíveis. 


\section{Lista de Gráficos}

Gráfico 1 - Taxa de erro médio de omissão por classe após a classificação supervisionada com atributos espectrais e textura.

Gráfico 2 - Erro de omissão médio por classe após a FASE 2. (resultado após 20 execuções)

Gráfico 3 - Erro de classificação global após a FASE 2. (resultado após 20 execuções)

Gráfico 4 - Erro de classificação global após a inclusão de conhecimento multitemporal de maneira CRISP. (resultado após 20 execuções) 82

Gráfico 5 - Evolução do algoritmo genético durante a sua evolução. 84

Gráfico 6 - Avaliação do erro de omissão após a inclusão da informação multitemporal nebulosa.

Gráfico 7 - Taxa de erro global após a classificação por inclusão de dados multitemporais nebulosos. 